\title{
SOME THEOREMS CONCERNING FUNCTION ALGEBRAS
}

\section{BY ERRETT BISHOP ${ }^{1}$}

Communicated by J. L. Kelley, November 21, 1958

In what follows, $C$ will be a compact Hausdorff space, and $\mathfrak{A}$ will be a uniformly closed separating algebra of continuous complexvalued functions on $C$. That is, sums, products, and complex multiples of elements of $\mathfrak{A}$ are in $\mathfrak{A}$, uniform limits of elements of $\mathfrak{A}$ are in $\mathfrak{A}$, and for distinct points $x$ and $y$ in $C$ there exists $f$ in $\mathfrak{A}$ with $f(x) \neq f(y)$. Silov (see [1]) has shown that there exists a smallest closed subset $B$ of $C$, called the Silov boundary of $\mathfrak{A}$, such that for each $f$ in $\mathfrak{A}$ there exists $x$ in $B$ with $|f(x)|=\|f\|$, where $\|f\|=\max \{|f(y)|: y \in C\}$. The following theorem generalizes this result, in case $C$ is metric.

TheOREM 1. Let $C$ be a compact metric space, and let $\mathfrak{A}$ be a uniformly closed separating algebra of continuous complex-valued functions on $C$. Then there exists a smallest subset $M$ of $C$, called the minimal boundary of $\mathfrak{A}$, such that for each $f$ in $\mathfrak{A}$ there exists $x$ in $M$ with $|f(x)|=\|f\|$. The set $M$ is equal to the set $M_{0}$ which is defined as follows:

$$
M_{0}=\{x: x \in C, \exists f \text { in } \mathfrak{A} \text { with }|f(x)|>|f(y)| \text { for all } y \neq x \text { in } C\} \text {. }
$$

The closure of $M$ is the Silov boundary of $\mathfrak{A}$.

The question of the topological structure of $M$ is answered by the following theorem.

Theorem 2. Let $\rho$ be the metric on $C$. For each positive integer $n$, let $U_{n}=\{x: x \in C, \exists f$ in $\mathfrak{A}$ with $\|f\| \leqq 1$,

$$
\left.|f(x)|>3 / 4, \text { and }|f(y)|<1 / 4 \text { for } \rho(x, y) \geqq n^{-1}\right\} .
$$

Then $\cap U_{n}=M$.

Since it is easy to see that $U_{n}$ is open, it follows that $M$ is a $G_{\delta}$.

It is known that every bounded linear functional $\phi$ on $\mathfrak{A}$ can be represented by a complex-valued Borel measure $\mu$ with $\|\mu\|=\|\phi\|$ on the Silov boundary $B$ of $\mathfrak{A}$. It is conjectured that $\mu$ can be taken to be a measure on the minimal bounary of $\mathfrak{A}$. The following theorem constitutes an important case of this conjecture.

TheOREM 3. Let $x$ be a point of $C$ which is not in the minimal boundary $M$ of $\mathfrak{A}$. Let $\mathfrak{A}$ contain the constant functions. Then there exists a non-negative Borel measure $\mu$ on $B-\{x\}$ of norm 1 such that $f(x)$ $=\int f d \mu$ for all $f$ in $\mathfrak{A}$.

1 This research was supported by the Sloan Foundation. 
As an application of these concepts, we have the following theorem.

THEOREM 4. Let $C$ be a compact subset of the complex plane without interior. Let $\mathfrak{A}$ be the algebra of all continuous functions on $C$ which are uniform limits of rational functions with poles in $-C$. Let $M$ be the minimal boundary of $\mathfrak{A}$. Let $\mathfrak{B}$ be the set of all continuous real functions on $C$ which are uniform limits of the real parts of rational functions with poles in $-C$. Let $N$ be the set

$$
\{x: x \in C, \exists f \text { in } \mathfrak{B} \text { with }|f(x)|>|f(y)| \text { for all } y \neq x \text { in } C\} .
$$

Then $M=N$ and the following conditions are equivalent:

(i) $\mathfrak{A}$ consists of all continuous functions on $C$,

(ii) $\mathfrak{B}$ consists of all continuous real functions on $C$,

(iii) $M=C$,

(iiii) $C-M$ has measure 0 .

Theorem 4 gives a necessary and sufficient condition on a compact subset $C$ of the complex plane that every continuous function on $C$ be uniformly approximable by rational functions. Mergelyan [2] has given sufficient conditions.

Added in proof. The conjecture preceding Theorem 3 has been proved.

\section{REFERENCES}

1. L. H. Loomis, Abstract harmonic analysis, New York, 1953.

2. S. N. Mergelyan, On the representation of functions by series of polynomials on closed sets, Amer. Math. Soc. Translations, no. 85, 1953.

University of California, Berkley 